\title{
Intraoperative Skin Injury
}

National Cancer Institute

\section{Source}

National Cancer Institute. Intraoperative Skin Injury. NCI Thesaurus. Code C78389.

Damage to the skin during a surgical procedure. 